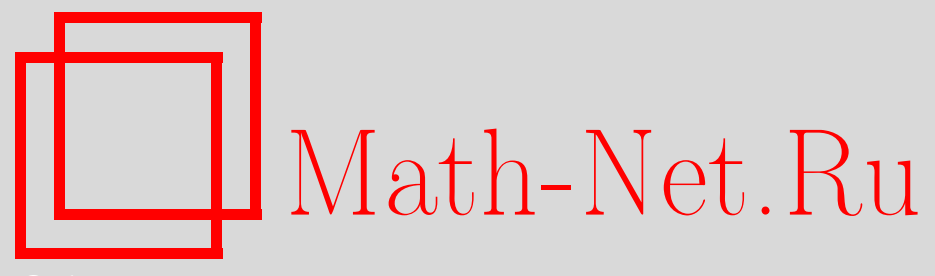

Е. В. Мурашкин, Ю. Н. Радаев, О согласовании ориентаций тензорных элементов площади в микрополярном континууме, погружаемом во внешнее плоское пространство, Вестн. Сам. гос. техн. унта. Сер. Физ.-мат. науки, 2021, номер 4, 776-786

DOI: https://doi.org/10.14498/vsgtu1883

Использование Общероссийского математического портала MathNet.Ru подразумевает, что вы прочитали и согласны с пользовательским соглашением http://www . mathnet.ru/rus/agreement

Параметры загрузки:

IP: 54.166 .219 .16

26 апреля 2023 г., 17:50:08

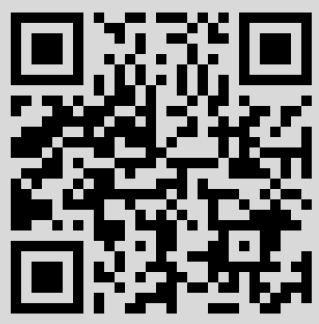


УДК 539.3

\title{
О согласовании ориентаций тензорных элементов площади в микрополярном континууме, погружаемом во внешнее плоское пространство
}

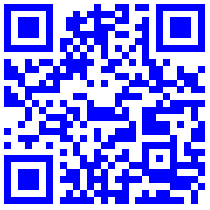

\section{Е. В. Мурашкин, Ю. Н. Радаев}

Институт проблем механики им. А. Ю. Ишлинского РАН, Россия, 119526, Москва, просп. Вернадского, 101, корп. 1.

\begin{abstract}
Аннотация
Рассматриваются проблемы согласования ориентаций реперов для микрополярного континуума, погруженного во внешнее плоское пространство. На основе понятия элементарного тензорного объема (площади) $M$-ячейки, описывается алгоритм сравнения и согласования внешних пространственных ориентаций $M$-ячеек. Рассматривается процесс непрерывного переноса реперных направлений, ассоциированных с $M$ ячейкой. В результате можно вести речь об ориентации самого микрополярного континуума и его границы. Ориентированный континуум играет важную роль в микрополярной теории упругости, корректное построение которой возможно только в рамках псевдотензорного формализма и ориентируемого многообразия. В особенности это касается теории гемитропных упругих сред. Обсуждается псевдотензорная формулировка теоремы Стокса.
\end{abstract}

Ключевые слова: относительный тензор, ориентирующий псевдоскаляр, микроповорот, перемещение, микрополярный гемитропный континуум.

Получение: 11 сентября 2021 г. / Исправление: 29 октября 2021 г. / Принятие: 22 ноября 2021 г. / Публикация онлайн: 24 декабря 2021 г.

\section{Краткое сообщение}

(C) Коллектив авторов, 2021

(c) СамГТУ, 2021 (составление, дизайн, макет)

(ㅇ) Кон Контент публикуется на условиях лицензии Creative Commons Attribution 4.0 International (https://creativecommons.org/licenses/by/4.0/deed.ru)

\section{Образец для цитирования}

М у рашк и н Е. В., Р а д а е в Ю. Н. О согласовании ориентаций тензорных элементов площади в микрополярном континууме, погружаемом во внешнее плоское пространство // Вестн. Сам. гос. техн. ун-та. Сер. Физ.-мат. науки, 2021. Т. 25, № 4. С. 776-786. https://doi.org/10.14498/vsgtu1883.

\section{Сведения об авторах}

Евгений Валерьевич Мурашкин (1) https://orcid.org/0000-0002-3267-4742

кандидат физико-математических наук; старший научный сотрудник; лаб. моделирования в механике деформируемого твердого тела; e-mail: evmurashkin@google.com

Юрий Николаевич Радаев (1) https://orcid.org/0000-0002-0866-2151

доктор физико-математических наук, профессор; ведущий научный сотрудник; лаб. моделирования в механике деформируемого твердого тела; e-mail:radayev@ipmnet.ru, y.radayev@gmail.com 
Введение. Наиболее распространенной математической моделью континуума является дифференцируемое многообразие $[1,2] .{ }^{1}$ В механике сплошных деформируемых сред обычно требуется, чтобы континуум был погружен во внешнее «плоское» пространство. ${ }^{2}$

На дифференцируемом многообразии часто приходится вводить дополнительные структуры. Например, риманова структура на многообразии позволяет говорить о целом классе дифференцируемых пространств [1,2], которые имеют важное прикладное значение. Подобные проблемы возникают при моделировании процессов деформирования материалов с микроструктурой, микрополярных сред, процессов аддитивного производства [3,4].

Другой важной особенностью, возникающей при моделировании поведения микрополярных материалов, является ориентируемость континуума и его границы. При формулировке интегральных теорем и законов механики континуума особое внимание надо уделять согласованию ориентаций реперных направлений элементарных ячеек внутри континуума и на его границе. Все базовые понятия, связанные с измерениями тензорного объема ячейки, ${ }^{3}$ требуют привлечения аппарата псевдотензорного исчисления и фундаментального понятия об ориентируемых многообразиях [5-11].

В ходе изложения вопросов, связанных с многомерной геометрией, будем следовать терминологии и идеям $[11,12]$. Минимальные сведения о тензорных элементах объема и площади можно найти в [8, см. приложение Дж. Л. Эриксена] и [11]. Вопросы применения алгебры псевдотензоров к задачам механики растущих тел и микрополярной теории упругости обсуждались в работах $[3,4,13-18]$.

В представленной работе рассмотрим проблемы согласования ориентаций реперов для микрополярного континуума, погруженного во внешнее плоское пространство. Опираясь на понятие элементарного тензорного объема (площади) $M$-ячейки, опишем алгоритм сравнения и согласования внешних пространственных ориентаций $M$-ячеек. Соответствующие рассмотрения подразумевают выбор ориентации репера, определяющего элемент объема (площади). Последнее обстоятельство имеет исключительное значение в микрополярных теориях упругости [19-21]. В особенности это касается теории гемитропных упругих сред $[3,13,14]$. Обсуждается псевдотензорная формулировка теоремы Стокса. ${ }^{4}$

\section{1. Тензорные элементы площади $M$-многообразия, погруженно-} го в $\boldsymbol{N}$-мерное пространство. В данной работе мы не будем воспроизводить определение и свойства псевдотензоров. Подробное изложение алгебры псевдотензоров можно найти в руководствах по тензорному анализу [7,9-11]

\footnotetext{
${ }^{1}$ Ориентируемое многообразие естественным образом появляется в теории микрополярной упругости.

${ }^{2}$ Особенно это актуально для механики растущих тел [3,4], когда растущее тело, вообще говоря, не допускает погружения в трехмерное пространство наблюдателя.

${ }^{3}$ Упомянутая процедура измерения и формирования тензорного объема ячейки приведена в работе [18].

${ }^{4} \mathrm{~B}$ существующей научной литературе указанная теорема обычно формулируется в терминах дифференциальных форм. В данной статье мы не будем пользоваться теорией дифференциальных форм. Более того, как хорошо известно, теория дифференциальных форм может быть переизложена в терминах антисимметричных псевдотензоров и ориентируемых многообразий.
} 


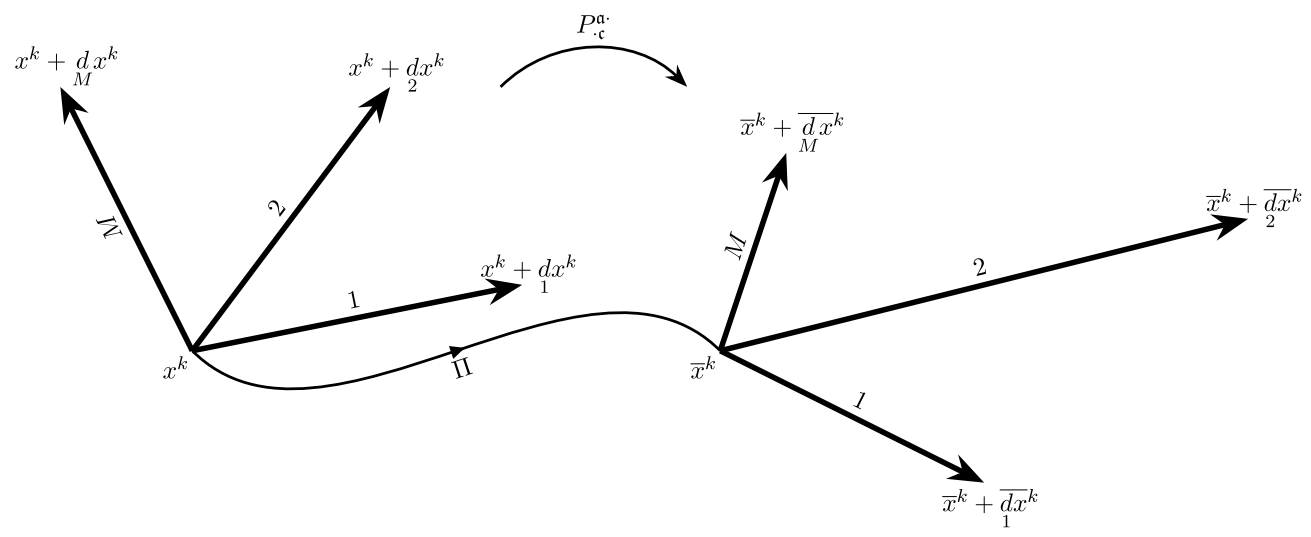

Реперные направления, ассоциированные с $M$-ячейками. Сравнение ориентаций осуществляется путем непрерывного переноса вдоль пути П одного репера к другому

[Reper directions associated with $M$-cells. Comparison of orientations is carried out by continuous transfer along the path $\Pi$ of one frame to another]

и в статьях $[3,14]$. В дальнейшем изложении сверху корневого символа относительного тензора в квадратных скобках будем отмечать его вес. Нулевой вес, присущий абсолютным тензорам, не отражается нами в обозначениях.

В $N$-мерном «плоском» пространстве выберем криволинейную систему координат $x^{k}(k=1,2, \ldots, N)$. Будем называть $M$-многообразием-многообразие (поверхность) математической размерности $M(M \leqslant N)$, погруженное в указанное внешнее пространство. Рассмотрим два репера с различными угловыми точками $x^{k}$ и $\bar{x}^{k}$ и концевыми точками $x^{k}+d_{\mathfrak{c}} x^{k}$ и $\bar{x}^{k}+\overline{d x}_{\mathfrak{c}}^{k}$, изображенными на рисунке. Тогда внешние координаты векторов первого и второго реперов будут $\underset{\mathfrak{c}}{d x^{k}}$ и $\underset{\mathfrak{c}}{d x} x^{k}$ соответственно. Очевидно, что всегда существует линейное преобразование одного репера к другому, действующее по формуле $[2,22]$

$$
\overline{d x} x_{\mathfrak{c}}^{k}=P_{\cdot \mathfrak{c}}^{\mathfrak{a} \cdot} d \underset{\mathfrak{a}}{d} x^{k}
$$

где $P_{\cdot \mathfrak{c}}^{\mathfrak{a} \cdot}$ - матрица перехода от старого базиса (репера) к новому $[2,22-24] .^{5}$ Опираясь на формулу (1), нетрудно сформулировать критерий сравнения ориентаций реперов $d x^{k}$ и $\overline{d x} k$. Для этого, во-первых, должен прежде всего существовать непрерывный путь П переноса одного $M$-репера в другой. Во-вторых, если $\operatorname{det}\left(P_{\cdot \mathfrak{c}}^{\mathfrak{a} \cdot}\right)>0$, то тогда считаем, что реперы имеют одинаковые ориентации или они коориентированы, а если $\operatorname{det}\left(P_{\cdot \mathfrak{c}}^{\mathfrak{a} \cdot}\right)<0$, то тогда реперы имеют противоположные ориентации. Более того, таким образом в каждой точке $M$-многообразия можно задать только два класса ориентаций реперов, непрерывно зависящих от точки многообразия.

Определим операцию непрерывного переноса репера из $M$ направлений вдоль пути П точки $x^{k}$ в точку $\bar{x}^{k}$ (см. рисунок) [25, с. 532]. Пусть имеется кусочно-гладкий путь П на $M$-многообразии, соединяющий две точки

\footnotetext{
${ }^{5}$ В руководствах по векторному анализу и конечномерным пространствам $[23,24]$ иногда вводится матрица перехода, транспонированная к (1). Мы следуем определению, данному в [22, с. 105].
} 
$x^{k}$ и $\bar{x}^{k}$. Переместим, непрерывно деформируя, репер из начальной точки в конечную так, чтобы векторы репера оставались линейно независимыми. Полученный репер можно сравнить с репером в конечной точке, если при этом ориентация репера не изменилась, т.е. матрица перехода в (1) не меняет знак, то многообразие является ориентируемым или двусторонним. В противном случае оно неориентируемо. Указанное обстоятельство говорит о том, что связное $M$-многообразие ориентируемо тогда и только тогда, когда параллельный перенос вдоль любого цикла внутри многообразия сохраняет ориентацию репера.

Ориентируемые многообразия имеют исключительное значение в микрополярных теориях механики континуума [19-21]. Ясно, что ориентация репера в точке микрополярного тела задается нумерацией реперных направлений. При перестановке двух номеров реперных направлений ориентация всего репера изменяется на противоположную, т.е. правоориентированный репер становится левоориентированным. В механике континуума ориентацию базисного репера удобно задавать фундаментальным ориентирующим скаляром $e[3,14]$. Его можно определить как косое произведение векторов ковариантного базиса $\underset{1}{\boldsymbol{\imath}}, \underset{2}{\boldsymbol{\imath}}, \ldots, \underset{N}{\boldsymbol{\imath}}$ в $N$-мерном пространстве $[12$, с. 63$]$

$$
\lceil\underset{1}{\boldsymbol{\imath}}, \underset{2}{\boldsymbol{\imath}}, \ldots, \underset{N}{\boldsymbol{\imath}}\rfloor=e
$$

В трехмерном пространстве $e$ определяется смешанным произведением базисных векторов

$$
e=\stackrel{[+1]}{e}=\lceil\underset{1}{\boldsymbol{\imath}}, \underset{2}{\boldsymbol{\imath}}, \underset{3}{\boldsymbol{\imath}}\rfloor=(\underset{1}{\boldsymbol{\imath}} \times \underset{2}{\boldsymbol{\imath}}) \cdot \underset{3}{\boldsymbol{\imath}} \text {. }
$$

Отметим, что фундаментальный ориентирующий псевдоскаляр позволяет легко преобразовывать псевдотензоры произвольного веса $W$ в абсолютные тензоры. Введем тензор $\mathbf{T}$ согласно

$$
\mathbf{T}=e^{-W^{[W]}} \stackrel{[}{\mathbf{T}}
$$

Сравнивая веса, приходим к заключению о том, что $\mathbf{T}$ является абсолютным тензором. В дальнейшем изложении у фундаментальных символов, таких как $\epsilon, e$ и $g$, указание на их вес будем опускать.

Отметим также широко используемую в механике и физике формулу

$$
e^{2}=g
$$

Пусть рассматриваемое дифференцируемое $M$-многообразие можно задать его Гауссовой параметризацией $u^{\alpha}(\alpha=1,2, \ldots, M)$

$$
x^{k}=x^{k}\left(u^{1}, u^{2}, \ldots, u^{M}\right) .
$$

В формуле $(2) x^{k}$ являются внешними координатами для $M$-многообразия, а $u^{\alpha}$ - внутренними.

Разобьем $M$-многообразие на систему $M$-ячеек ( $M$-cell). Каждая $M$-ячейка задается угловым репером, который характеризуется угловой вершиной 
(с внешними координатами $x^{k}$ и внутренними координатами $u^{\alpha}$ ) и концевыми точками репера, имеющими внутренние координаты

$$
u^{\alpha}+\underset{\mathfrak{c}}{d u^{\alpha}}, \quad \alpha=1,2, \ldots, M
$$

и внешние координаты

$$
x^{k}+\underset{\mathfrak{c}}{d x^{k}}, \quad k=1,2, \ldots, N
$$

где индекс шрифта «фрактур» с нумерует реперные направления $(\mathfrak{c}=1,2, \ldots$, $M)$. С внешней (пространственной) точки зрения направления рассматриваемого репера задаются абсолютными контравариантными векторами

$$
\underset{1}{d x^{k}}, \underset{2}{d x^{k}}, \ldots, \underset{M}{d} x^{k}, \quad k=1,2, \ldots, N
$$

Тензорный элемент объема $M$-ячейки (the tensor element of area $[8$, см. приложение Дж. Л. Эриксена с. 816] $)^{6}$ определим согласно формуле

$$
d \tau^{i_{1} i_{2} \ldots i_{M}}=M ! d x_{2}^{\left[i_{1}\right.} \underset{2}{d} x^{i_{2}} \cdots \underset{M}{d} x^{\left.i_{M}\right]} .
$$

Здесь в квадратные скобки заключены индексы, по которым выполняется альтернирование.

Учитывая формулу для дифференциалов внешних координат вдоль реперных направлений $M$-ячейки

$$
\underset{\mathfrak{b}}{d x^{k}}=\left(\partial_{\alpha} x^{k}\right) \underset{\mathfrak{b}}{d u^{\alpha}}
$$

соотношение (3) можно записать в виде [11, с. 256-257]

$$
d \tau^{i_{1} i_{2} \ldots i_{M}}=\delta_{\gamma_{1} \gamma_{2} \ldots \gamma_{M}}^{\alpha_{1} \alpha_{2} \ldots \alpha_{M}} \partial_{\alpha_{1}} x^{i_{1}} \partial_{\alpha_{2}} x^{i_{2}} \cdots \partial_{\alpha_{M}} x_{1}^{i_{M}} \underset{1}{d} u_{2}^{\gamma_{1}} d u^{\gamma_{2}} \cdots \underset{M}{d} u^{\gamma_{M}}
$$

где $\delta_{\gamma_{1} \gamma_{2} \ldots \gamma_{M}}^{\alpha_{1} \alpha_{2} \ldots \alpha_{M}}$ - обобщенные дельты Кронекера. Воспользовавшись известной из псевдотензорного исчисления формулой для контравариантных символов перестановок $\epsilon^{\alpha_{1} \alpha_{2} \ldots \alpha_{M}}$

$$
\epsilon^{\alpha_{1} \alpha_{2} \ldots \alpha_{M}}=\delta_{12 \ldots M}^{\alpha_{1} \alpha_{2} \ldots \alpha_{M}}
$$

получим

$$
d \tau^{i_{1} i_{2} \ldots i_{M}}=\epsilon^{\alpha_{1} \alpha_{2} \ldots \alpha_{M}} \partial_{\alpha_{1}} x^{i_{1}} \partial_{\alpha_{2}} x^{i_{2}} \cdots \partial_{\alpha_{M}} x^{i_{M}} \operatorname{det}\left(\underset{\mathfrak{b}}{d} u^{\gamma}\right)
$$

Если элементарные $M$-ячейки нарезаны с помощью координатных поверхностей $u^{\alpha}=c^{\alpha}$, то тогда справедливо соотношение

$$
\operatorname{det}\left(d u^{\gamma}\right)=d u^{1} d u^{2} \cdots d u^{M}
$$

и для случая $M=N$ получим

$$
d \tau^{i_{1} i_{2} \ldots i_{M}}=\stackrel{[-1]}{d} \tau^{12 \ldots M} \epsilon^{i_{1} i_{2} \ldots i_{M}}
$$

\footnotetext{
${ }^{6}$ Согласно более архаичной терминологии (см. [11, с. 255]) можно также использовать термин "протяженность $M$-ячейки" (the extension of the $M$-cell).
} 
$[-1]$

где $d \tau 12 \ldots M-$ естественный элемент объема, представляющий собой псевдоскаляр веса -1 , который определяется следующим образом:

$$
{ }^{[-1]} \tau^{12 \ldots M}=\operatorname{det}\left(\partial_{\alpha} x^{k}\right) d u^{1} d u^{2} \cdots d u^{M}=d x^{1} d x^{2} \cdots d x^{M} .
$$

С помощью псевдоскаляра $d \tau$ и фундаментального ориентирующего скаляра $e$ можно образовать абсолютный скаляр $d \tau$, являющийся инвариантным элементом объема

$$
d \tau=e d \tau^{[-1]} 12 \ldots M
$$

2. Теорема Стокса. Важную роль в механике континуума, особенно при формулировке законов сохранения, играют интегральные теоремы. Запишем инвариантный интеграл на $M$-многообразии

$$
\int A_{i_{1} i_{2} \ldots i_{M}} d \tau^{i_{1} i_{2} \ldots i_{M}}
$$

где $A_{i_{1} i_{2} \ldots i_{M}}$ - абсолютный ковариантный тензор валентности $M$. Интеграл (4) является абсолютным инвариантом.

Рассмотрим граничное $(M-1)$-многообразие $\partial$ к $M$-многообразию, заданное своей Гауссовой параметризацией

$$
x^{k}=x^{k}\left(\widetilde{u}^{1}, \widetilde{u}^{2}, \ldots, \widetilde{u}^{M-1}\right),
$$

тогда $(M-1)$-ячейка на границе $\partial$ будет определяться репером $\widetilde{\partial}_{1} x^{k}, \widetilde{\partial}_{2} x^{k}, \ldots$, $\widetilde{\partial}_{M-1} x^{k}$, где $\widetilde{\partial}_{i}=\partial / \partial \widetilde{u}^{i}$. Зададим на границе $\partial$ векторное поле $S^{k}$, направленное вовне по отношению к $M$-многообразию. Согласуем (arrange) параметризации (2) и (5) так, чтобы ориентации систем векторов так $\widetilde{\partial}_{1} x^{k}, \widetilde{\partial}_{2} x^{k}, \ldots$, $\widetilde{\partial}_{M-1} x^{k}, S^{k}$ и $\partial_{1} x^{k}, \partial_{2} x^{k}, \ldots, \partial_{M} x^{k}$ были одинаковыми, т.е. матрица перехода от одной системы векторов к другой имела бы положительный детерминант.

Для любого дифференцируемого ковариантного абсолютного тензорного поля $A_{i_{1} i_{2} \ldots i_{M-1}}$ можно сформулировать теорему Стокса [11, p. 269]:

$$
\int \partial_{i_{M}} A_{i_{1} i_{2} \ldots i_{M-1}} d \tau^{i_{1} i_{2} \ldots i_{M}}=\oint_{\partial} A_{i_{1} i_{2} \ldots i_{M-1}} d \tau^{i_{1} i_{2} \ldots i_{M-1}},
$$

где $d \tau^{i_{1} i_{2} \ldots i_{M}}$ вычисляется с использованием параметризации $u^{1}, u^{2}, \ldots, u^{M}$, а $d \tau^{i_{1} i_{2} \ldots i_{M-1}}-$ с использованием параметризации $\widetilde{u}^{1}, \widetilde{u}^{2}, \ldots, \widetilde{u}^{M-1}$.

Подынтегральное выражение для $A_{i_{1} i_{2} \ldots i_{M-1}}$ можно преобразовать следующим образом:

$$
\partial_{i_{M}} A_{i_{1} i_{2} \ldots i_{M-1}} d \tau^{i_{1} i_{2} \ldots i_{M}}=\partial_{\left[i_{M}\right.} A_{\left.i_{1} i_{2} \ldots i_{M-1}\right]} d \tau^{i_{1} i_{2} \ldots i_{M}}=\nabla_{\left[i_{M}\right.} A_{\left.i_{1} i_{2} \ldots i_{M-1}\right]} d \tau^{i_{1} i_{2} \ldots i_{M}} .
$$

Заключение. В статье рассматриваются проблемы согласования ориентаций реперов для микрополярного континуума, погруженного во внешнее плоское пространство. 
1. На основе понятия элементарного тензорного объема (площади) $M$ ячейки описывается алгоритм сравнения и согласования внешних пространственных ориентаций $M$-ячеек.

2. Рассматривается процесс непрерывного переноса реперных направлений, ассоциированных с $M$-ячейкой. В результате можно вести речь об ориентации самого микрополярного континуума и его границы.

3. Ориентированный континуум играет важную роль в микрополярной теории упругости, корректное построение которой возможно только в рамках псевдотензорного формализма и ориентируемого многообразия. В особенности это касается теории гемитропных упругих сред.

4. Обсуждается псевдотензорная формулировка теоремы Стокса.

Конкурирующие интересы. Конкурирующих интересов не имеем.

Авторский вклад и ответственность. Все авторы принимали участие в разработке концепции статьи; все авторы сделали эквивалентный вклад в подготовку публикации. Авторы несут полную ответственность за предоставление окончательной рукописи в печать. Окончательная версия рукописи была одобрена всеми авторами.

Финансирование. Работа выполнена в рамках государственного задания (№ госрегистрации АААА-А20-120011690132-4) и при поддержке Российского фонда фундаментальных исследований проекты № 19-51-60001, № 20-01-00666.

\section{Библиографический список}

1. Eisenhart L. P. Riemannian Geometry. Princeton, N.J.: Princeton Univ., 1967. vii +306 pp.

2. Рашевский П. К. Риманова геометрия и тензорный анализ. М.: Наука, 1967. 664 с.

3. Murashkin E. V., Radayev Yu. N. On a micropolar theory of growing solids // Vestn. Samar. Gos. Tekhn. Univ., Ser. Fiz.-Mat. Nauki [J. Samara State Tech. Univ., Ser. Phys. Math. Sci.], 2020. vol. 24, no. 3. pp. 424-444. https://doi.org/10.14498/vsgtu1792.

4. Murashkin E. V., Radayev Y. N. On a differential constraint in asymmetric theories of the mechanics of growing solids // Mech. Solids, 2019. vol.54, no. 8. pp. 1157-1164. https:// doi.org/10.3103/S0025654419080053.

5. Veblen O., Thomas T. Y. Extensions of relative tensors // Trans. Amer. Math. Soc., 1924. vol. 26, no. 3. pp. 373-377. https://doi.org/10.1090/s0002-9947-1924-1501284-6.

6. Veblen O. Invariants of Quadratic Differential Forms / Cambridge Tracts in Mathematics and Mathematical Physics. vol. 24. Cambridge: Cambridge Univ. Press, 1927. viii +102 pp.

7. Gurevich G. B. Foundations of the Theory of Algebraic Invariants. Groningen: P. Noordhoff, 1964. viii +429 pp.

8. Truesdell C., Toupin R. The Classical Field Theories / Principles of Classical Mechanics and Field Theory / Encyclopedia of Physics, III/1; ed. S. Flügge. Berlin, Göttingen, Heidelberg: Springer, 1960. pp. 226-902. https://doi.org/10.1007/978-3-642-45943-6_2.

9. Schouten J. A. Tensor Analysis for Physicists. Oxford: Clarendon Press, 1954. xii +277 pp.

10. Sokolnikoff I. S. Tensor Analysis: Theory and Applications to Geometry and Mechanics of Continua/ Applied Mathematics Series. New York: John Wiley \& Sons Inc, 1964. xii +361 pp.

11. Synge J. L., Schild A. Tensor Calculus / Dover Books on Advanced Mathematics. vol. 5. New York: Courier Corporation, 1978. ix +324 pp.

12. Розенфельд Б. А. Многомерные пространства. М.: Наука, 1966. 647 с.

13. Kovalev V. A., Murashkin E. V., Radayev Yu. N. On the Neuber theory of micropolar elasticity. A pseudotensor formulation//Vestn. Samar. Gos. Tekhn. Univ., Ser. Fiz.-Mat. Nauki [J. Samara State Tech. Univ., Ser. Phys. Math. Sci.], 2020. vol. 24, no. 4. pp. 752-761. https://doi.org/10.14498/vsgtu1799. 
14. Радаев Ю. Н., Мурашкин Е. В. Псевдотензорная формулировка механики гемитропных микрополярных сред// Проблемы прочности и пластичности, 2020. Т. 82, № 4 . C. 399-412. https://doi.org/10.32326/1814-9146-2020-82-4-399-412.

15. Мурашкин Е. В., Радаев Ю. Н. Об одном обобщении алгебраической теории Гамильтона-Кэли // Изв. РАН. МТT, 2021. №6. С. 130-138. https://doi.org/10.31857/ S0572329921060106.

16. Мурашкин Е. В., Радаев Ю. Н. Об одном псевдотензорном обобщении связывающих двусторонних граничных условий Югонио-Адамара // Вестн. ЧГПУ им. И.Я. Яковлева. Сер. Механика предельного состояния, 2021. № 2(48). C. 104-114. https://doi . org/10.37972/chgpu.2021.48.2.013.

17. Мурашкин Е. В., Радаев Ю. Н. Прямые, инверсные и зеркальные волновые моды связанных волн перемещений и микровращений в гемитропных микрополярных средах // Вестн. ЧГПУ им. И.Я. Яковлева. Сер. Механика предельного состояния, 2021. № 2(48). C. 115-127. https ://doi .org/10.37972/chgpu .2021.48.2.014.

18. Мурашкин Е. В., Радаев Ю. Н. К теории ориентированных тензорных элементов площади микрополярного континуума, погруженного во внешнее плоское пространство // Изв. РАН. МТТ, 2022. № 2 (в печати).

19. Nowacki W. Theory of Micropolar Elasticity. Course held at the Department for Mechanics of Deformable Bodies, July 1970, Udine/ International Centre for Mechanical Sciences. Courses and Lectures. vol. 25. Wien, New York: Springer, 1972. 286 pp. https://doi.org/ 10.1007/978-3-7091-2720-9.

20. Nowacki W. Theory of Asymmetric Elasticity. Oxford: Pergamon Press, 1986. viii +383 pp.

21. Besdo D. Ein Beitrag zur nichtlinearen Theorie des Cosserat-Kontinuums [A contribution to the nonlinear theory of the Cosserat-continuum] // Acta Mechanica, 1974. vol. 20, no. 1. pp. 105-131 (In German). https://doi.org/10.1007/BF01374965.

22. Шилов Г. Е. Введение в теорию линейных пространств. М.: ГИТТЛ, 1952. 384 с.

23. Сушкевич А. К. Основы высшей алгебры. М.: ОНТИ, 1937. 476 с.

24. Lagally M. Vorlesungen über Vektor-Rechnung [Vector Calculus]. Leipzig: Akademische Verlagsgesellschaft, 1928. xvii +358 pp. (In German)

25. Дубровин Б. А., Новиков С. П., Фоменко А. Л. Современная геометрия. М.: Наука, 1979. $760 \mathrm{c.}$ 


\title{
MSC: 15A72, 53A45, 74D05
}

\section{On a ordering of area tensor elements orientations in a micropolar continuum immersed in an external plane space}

\author{
E. V. Murashkin, Yu. N. Radayev \\ Ishlinsky Institite for Problems in Mechanics, Russian Academy of Sciences, \\ 101-1, pr. Vernadskogo, Moscow, 119526, Russian Federation.
}

\begin{abstract}
The paper deals with the problems of ordering the reper orientations for a micropolar continuum immersed in an external plane space. Based on the concept of an elementary tensor volume (area) $M$-cells, an algorithm for comparing and matching external spatial orientations of $M$-cells is proposed. The process of continuous transfer of reper directions associated with a $M$-cell is considered. As a result, we can talk about the orientation of micropolar continuum itself and its boundary. The oriented continuum plays an important role in micropolar elasticity. This is especially true for the theory of hemitropic elastic media. The pseudotensor formulation of Stokes' theorem is discussed.
\end{abstract}

Keywords: relative tensor, orienting pseudoscalar, microrotation, displacement, micropolar hemitropic continuum.

Received: $11^{\text {th }}$ September, $2021 /$ Revised: $29^{\text {th }}$ October, 2021

Accepted: $22^{\text {nd }}$ November, $2021 /$ First online: $24^{\text {th }}$ December, 2021

Competing interests. The authors declare no conflicts of interests.

Authors' contributions and responsibilities. Each author has participated in the article concept development; the authors contributed equally to this article. The authors are absolutely responsible for submit the final manuscript to print. Each author has approved the final version of manuscript.

\section{Short Communication}

(C) Authors, 2021

(C) Samara State Technical University, 2021 (Compilation, Design, and Layout)

() (1) The content is published under the terms of the Creative Commons Attribution 4.0 International License (http://creativecommons.org/licenses/by/4.0/)

\section{Please cite this paper in press as:}

Murashkin E. V., Radayev Yu. N. On a ordering of area tensor elements orientations in a micropolar continuum immersed in an external plane space, Vestn. Samar. Gos. Tekhn. Univ., Ser. Fiz.-Mat. Nauki [J. Samara State Tech. Univ., Ser. Phys. Math. Sci.], 2021, vol. 25, no. 4, pp. 776-786. https://doi.org/10.14498/vsgtu1883 (In Russian).

\section{Authors' Details:}

Evgenii V. Murashkin (10) https://orcid.org/0000-0002-3267-4742

Cand. Phys. \& Math. Sci., PhD, MD; Senior Researcher; Lab. of Modeling in Solid Mechanics; e-mail: evmurashkin@gmail.com

Yuri N. Radayev (iD https://orcid.org/0000-0002-0866-2151

D.Sc. (Phys. \& Math. Sci.), Ph.D., M.Sc., Professor; Leading Researcher; Lab. of Modeling in Solid Mechanics; e-mail: radayev@ipmnet.ru, y.radayev@gmail.com 
Funding. This study was supported by the Ministry of Science and Higher Education of the Russian Federation (State Registration Number AAAA-A20-120011690132-4) and by the Russian Foundation for Basic Research (projects nos. 18-01-00844, 20-01-00666).

\section{References}

1. Eisenhart L. P. Riemannian Geometry. Princeton, N.J., Princeton Univ., 1967, vii +306 pp.

2. Raschewski P. K. Riemannsche Geometrie und Tensoranalysis [Riemannian Geometry and Tensor Analysis]. Frankfurt am Main, Verlag Harri Deutsch, 1995, 606 pp. (In German)

3. Murashkin E. V., Radayev Yu. N. On a micropolar theory of growing solids, Vestn. Samar. Gos. Tekhn. Univ., Ser. Fiz.-Mat. Nauki [J. Samara State Tech. Univ., Ser. Phys. Math. Sci.], 2020, vol. 24, no. 3, pp. 424-444. https://doi.org/10.14498/vsgtu1792.

4. Murashkin E. V., Radayev Y. N. On a differential constraint in asymmetric theories of the mechanics of growing solids, Mech. Solids, 2019, vol. 54, no. 8, pp. 1157-1164. https://doi. org/10.3103/S0025654419080053.

5. Veblen O., Thomas T. Y. Extensions of relative tensors, Trans. Amer. Math. Soc., 1924, vol. 26, no. 3, pp. 373-377. https://doi.org/10.1090/s0002-9947-1924-1501284-6.

6. Veblen O. Invariants of Quadratic Differential Forms, Cambridge Tracts in Mathematics and Mathematical Physics, vol. 24. Cambridge, Cambridge Univ. Press, 1927, viii +102 pp.

7. Gurevich G. B. Foundations of the Theory of Algebraic Invariants. Groningen, P. Noordhoff, 1964, viii +429 pp.

8. Truesdell C., Toupin R. The Classical Field Theories, In: Principles of Classical Mechanics and Field Theory, Encyclopedia of Physics, III/1; ed. S. Flügge. Berlin, Göttingen, Heidelberg, Springer, 1960, pp. 226-902. https://doi.org/10.1007/978-3-642-45943-6_2.

9. Schouten J. A. Tensor Analysis for Physicists. Oxford, Clarendon Press, 1954, xii +277 pp.

10. Sokolnikoff I. S. Tensor Analysis: Theory and Applications to Geometry and Mechanics of Continua, Applied Mathematics Series. New York, John Wiley \& Sons Inc, 1964, xii +361 pp.

11. Synge J. L., Schild A. Tensor Calculus, Dover Books on Advanced Mathematics, vol. 5. New York, Courier Corporation, 1978, ix +324 pp.

12. Rozenfel'd B. A. Mnogomernye prostranstva [Multidimensional Spaces]. Moscow, Nauka, 1966, 648 pp. (In Russian)

13. Kovalev V. A., Murashkin E. V., Radayev Yu. N. On the Neuber theory of micropolar elasticity. A pseudotensor formulation, Vestn. Samar. Gos. Tekhn. Univ., Ser. Fiz.-Mat. Nauki [J. Samara State Tech. Univ., Ser. Phys. Math. Sci.], 2020, vol. 24, no. 4, pp. 752-761. https://doi.org/10.14498/vsgtu1799.

14. Radayev Yu. N., Murashkin E. V. Pseudotensor formulation of the mechanics of hemitropic micropolar media, Problems of Strength and Plasticity, 2020, vol. 82, no. 4, pp. 399-412 (In Russian). https://doi.org/10.32326/1814-9146-2020-82-4-399-412.

15. Radayev Yu. N., Murashkin E. V. A generalization of the algebraic theory of Hamilton-Cayley, Mech. Solids, 2021, vol.56, no. 6 (to appear).

16. Murashkin E. V., Radayev Yu. N. On a pseudotensor generalization of the HugoniotHadamard linking boundary conditions, Vestn. I. Yakovlev Chuvach State Pedagogical Univ. Ser. Mechanics of a Limit State, 2021, no. 2(48), pp. 104-114 (In Russian). https://doi. org/10.37972/chgpu.2021.48.2.013.

17. Murashkin E. V., Radayev Yu. N. Direct, inverse and mirror wave modes of coupled displacements and microrotations monochromatic plane waves in hemitropic micropolar media, Vestn. I. Yakovlev Chuvach State Pedagogical Univ. Ser. Mechanics of a Limit State, 2021, no. 2(48), pp. 115-127 (In Russian). https://doi.org/10.37972/chgpu.2021.48.2.014.

18. Radayev Yu. N., Murashkin E. V. On the theory of oriented tensor elements of the area of a micropolar continuum immersed in an external flat space, Mech. Solids, 2022, vol. 57, no. 2 (to appear).

19. Nowacki W. Theory of Micropolar Elasticity. Course held at the Department for Mechanics of Deformable Bodies, July 1970, Udine, International Centre for Mechanical Sciences. 
Courses and Lectures, vol. 25. Wien, New York, Springer, 1972, 286 pp. https://doi.org/ 10.1007/978-3-7091-2720-9.

20. Nowacki W. Theory of Asymmetric Elasticity. Oxford, Pergamon Press, 1986, viii +383 pp.

21. Besdo D. Ein Beitrag zur nichtlinearen Theorie des Cosserat-Kontinuums [A contribution to the nonlinear theory of the Cosserat-continuum], Acta Mechanica, 1974, vol. 20, no. 1, pp. 105-131 (In German). https://doi.org/10.1007/BF01374965.

22. Shilov G. E. An Introduction to the Theory of Linear Spaces, Dover Books on Advanced Mathematics. New York, Dover Publ., 1974, ix +310 pp.

23. Sushkevich A. K. Osnovy vysshei algebry [Fundamentals of Higher Algebra]. Moscow, ONTI, 1937, 476 pp. (In Russian)

24. Lagally M. Vorlesungen über Vektor-Rechnung [Vector Calculus]. Leipzig, Akademische Verlagsgesellschaft, 1928, xvii +358 pp. (In German)

25. Dubrovin B. A., Novikov S. P., Fomenko A. L. Sovremennaia geometriia [Modern Geometry]. Moscow, Nauka, 1979, 760 pp. (In Russian) 